\title{
TRATAMENTO DA DEPRESSÃO EM CRIANÇAS E ADOLESCENTES COM O USO DA FLUOXETINA
}

\section{TREATMENT OF DEPRESSION IN CHILDREN AND ADOLESCENTS WITH THE USE OF FLUOXETINE}

\author{
Fabrícia Guimarães Gonçalves \\ Bacharel em Farmácia. Alfa - Faculdade de Almenara, Almenara-MG \\ Brasil. \\ Jamila Pereira Antunes Luz \\ Bacharel em Farmácia. Alfa - Faculdade de Almenara, Almenara-MG \\ Brasil. \\ Luiza Gobira Lacerda \\ Especialista. Alfa - Faculdade de Almenara, Almenara-MG Brasil. \\ Viviane Amaral Toledo Coelho \\ Doutora. vivianeatc@yahoo.com.br. Almenara-MG \\ Virginia Torres Alves \\ Especialista. Alfa - Faculdade de Almenara, Almenara-MG, Brasil.
}

\section{RESUMO}

Objetivo: verificar a importância da coleta do PSA sobre a prevenção do câncer de próstata. Metodologia: Trata-se de uma revisão sistemática da literatura bibliográfica de artigos científicos publicados entre os anos de 2004 a 2018. A pesquisa foi norteada pelas bases de dados eletrônicos: Scientific Eletronic Library Online (Scielo), Portal Regional BVS e Google Acadêmico. Para critérios de inclusão dos artigos resultantes 
foram adotados parâmetros como; relevância ao tema, artigos científicos e estudos escritos em português e inglês, atualidade das informações, ou conteúdo de anos anteriores se pertinentes. Foram utilizados os seguintes Descritores em Ciências da Saúde (DeCS): "câncer de próstata"; "prevenção e controle" e "epidemiologia" “diagnóstico". Resultados: De acordo com o Instituto Nacional de Câncer (INCA) o número de casos novos de Câncer de Próstata (CP) estimados no ano de 2008 para o Brasil foi de 49.530, o que corresponderia a um risco estimado de 52 casos novos para cada100 mil homens. Se manifesta principalmente acima dos 50 anos de idade e, com o aumento da expectativa de vida, é considerado muito importante nos dados apresentados de mortalidade entre homens, representando um sério problema de saúde pública. Considerações finais: Com essa revisão podemos analisar que o exame PSA é importantíssimo, porém não tanto quando associado ao toque retal e o exame de ultrassom transretal da próstata com biópsia. Visto que o PSA é altamente sensível, porém não é câncer-específico e a maioria dos homens com PSA elevado não tem CAP.

Palavras-chave: Câncer de próstata. Prevenção. Controle. Epidemiologia. Diagnóstico.

\section{ABSTRACT}

Objective: To verify the importance of PSA collection on prostate cancer prevention. Methodology: This is a systematic review of the bibliographic literature of scientific articles published between 2004 and 2018. The research was guided by electronic databases: Scientific Electronic Library Online (Scielo), Regional Portal VHL and Google Scholar. For inclusion criteria of the resulting articles were adopted parameters such as; relevance to the topic, scientific articles and studies written in Portuguese and English, timeliness of information, or content from previous years if pertinent. The following Health Sciences Descriptors (DeCS) were used: "prostate cancer"; "Prevention and control" and "epidemiology" "diagnosis". Results: According to the National Cancer Institute (INCA) the estimated number of new cases of prostate cancer (PC) in 2008 for Brazil was 49,530, which would correspond to an estimated risk of 52 
new cases for every 100. A thousand men. It manifests mainly above 50 years of age and, with increasing life expectancy, is considered very important in the reported mortality data among men, representing a serious public health problem. Final considerations: With this review we can analyze that the PSA exam is very important, but not so much when associated with rectal touch and transrectal prostate ultrasound exam with biopsy. Since PSA is highly sensitive, it is not cancer-specific and most men with high PSA do not have CAP.

Keywords: Prostate Cancer. Prevention. Control. Epidemiology. Diagnosis.

\section{INTRODUÇÃO}

A próstata se apresenta como a glândula do sistema reprodutor masculino que se encontra abaixo da bexiga, possuindo volume e aparência semelhante a uma castanha e é responsável por produzir o líquido seminal. Sabe-se que a próstata pode ser acometida pelas patologias a seguir destacadas: Hiperplasia Benigna Prostática, Prostatite e Câncer de Próstata (FERNANDES et al., 2014).

A neoplasia maligna da próstata conhecida popularmente como o câncer da próstata $(\mathrm{CaP})$ é o segundo tipo de câncer com maior incidência no mundo e o quinto em taxa de mortalidade. No Brasil, é o de maior incidência, excluindo os casos de câncer de pele, sendo, o segundo tipo de câncer de maior mortalidade. (RIBEIRO et al., 2015).

No Brasil, o CaP é o mais incidente em todas as regiões do país, com estimativa para 2016/2017 de cerca de 61 mil casos. Os estados brasileiros que apresentam maiores números de casos novos são Paulo, Rio Grande do Sul, Rio de Janeiro, Minas Gerais e Paraná. (MELO; TEIXEIRA; CARVALHO, 2013).

O PSA ainda é o marcador mais empregado no rastreamento e no acompanhamento do CaP. PSA é uma sigla em inglês do termo Prostate Specific Antigen e significa antígeno prostático específico. (FERNANDES et al., 2014).

Segundo Ascari et al., (2014) o PSA é um importante marcador utilizado para detectar, determinar o estágio e monitorar a hiperplasia benigna e o $\mathrm{CaP}$, bem como 
utilizá-lo para diferenciar as patologias prostáticas, benignas e malignas, e também evitar biópsias desnecessárias, sabendo-se que seus níveis aumentam conforme a idade mais avançada.

Salienta-se que na dosagem de PSA deve-se levar em consideração a história clínica do paciente, idade, uso de medicação e volume prostático. Não existe um valor de referência específico para a dosagem do PSA, apesar de muitos autores preconizar em valores acima de $10 \mathrm{ng} / \mathrm{mL}$ como sugestivo de CaP. É importante destacar que quanto maior for a elevação do PSA, maior é a chance de existir metástase (GUEDES; JUNIOR, 2014).

O homem resiste em realizar o exame de toque retal por preconceito, mas também, por falta de informação. Por isso, acredita-se que o conhecimento sobre a importância desse exame possa sensibilizar os homens a realizá-lo e a repeti-lo sem restrição.

\section{METODOLOGIA}

Trata-se de uma revisão sistemática da literatura, com a seguinte questão norteadora: quais as principais orientações acerca da prevenção primária do câncer de próstata?

Diante da pergunta, foi executado o levantamento bibliográfico de artigos científicos publicados entre os anos de 2004 a 2018. Após a fase de identificação os conteúdos serão revisados, por meio de leitura seletiva, para se definir quais representarão a etapa determinante para escolha do material que consistirá em fonte bibliográfica para a pesquisa.

A pesquisa norteará pelas bases de dados eletrônicos: Scientific Eletronic Library Online (Scielo), Portal Regional BVS e Google Acadêmico. Para critérios de inclusão dos artigos resultantes foram adotados parâmetros como; relevância ao tema, artigos científicos e estudos escritos em português e inglês, atualidade das informações, ou conteúdo de anos anteriores se pertinentes. Foram utilizados os seguintes Descritores 
em Ciências da Saúde (DeCS): "câncer de próstata"; "prevenção e controle" e “epidemiologia" "diagnóstico". Desta forma, a pesquisa abrangeu um total de 21 conteúdos estudados, onde através leitura criteriosa, foram selecionados.

\section{CÂNCER DE PRÓSTATA}

O câncer é um tumor ou neoplasia ocasionada por uma ruptura dos mecanismos regulares da multiplicação celular, sem que seja necessário ao tecido, uma célula começa a crescer e dividir-se desordenadamente, insensíveis aos mecanismos reguladores normais. Dessa forma o $\mathrm{CaP}$ é o câncer mais comum no homem e sua incidência tende a crescer com o aumento da expectativa de vida, sendo raro em homens antes dos 50 anos de idade. Alguns fatores de ricos foram identificados como idade, raça, etnia e história familiar de câncer pai ou irmão (FERNANDES et al., 2014).

Sendo assim pode-se caracterizar a próstata como um órgão pélvico exclusivo do homem. Situa-se logo abaixo da bexiga envolvida em toda sua extensão pela uretra e tem como função a produção de um líquido leitoso e alcalino que ajuda na formação do sêmen (BACARIN; OLIVEIRA, 2018).

Segundo Ascari et al. (2014) o CaP em seus estágios iniciais raramente produz sintomas. Os sintomas que se desenvolvem devido à obstrução urinária acontecem tardiamente na doença. Esse câncer tende a variar em sua evolução quando a neoplasia é suficientemente grande para avançar sobre o colo vesical, ocorrendo sinais e sintomas de obstrução urinária, dificuldade e frequência de micção, retenção urinário, tamanho e força de jato. Os outros sintomas podem incluir o sangue na urina ou sêmen e ejaculação dolorosa. A hematúria pode resultar quando o câncer invade a uretra ou bexiga, podendo gerar metástase para ossos e linfonodos. Quando o CaP é detectado precocemente, a probabilidade de cura é alta. Todo homem com mais de 40 anos de idade deve fazer o exame retal como parte de seus exames regulares de saúde. 


\section{EPIDEMIOLOGIA DO CÂNCER DE PRÓSTATA.}

Atualmente no Brasil, pode-se observar um aumento das taxas de incidência de $\mathrm{CaP}$ ao longo dos anos. É o tumor mais frequente no sexo masculino, e considerado o segundo câncer mais comum na população masculina no mundo, com aproximadamente 70\% dos casos diagnosticados em regiões mais desenvolvidas (ASCARI et al., 2014).

Aspectos étnicos e geográficos também são fatores de risco. $\mathrm{O} \mathrm{CaP}$ é aproximadamente duas vezes mais comum em homens negros se comparados aos brancos. Os estadunidenses, jamaicanos e caribenhos com ascendência africana apresentam as mais altas taxas de incidência do $\mathrm{CaP}$ do mundo, o que pode ser atribuído, em parte, à hereditariedade (cerca de 5\% a 10\%). Apesar disso, é possível que essa diferença entre negros e brancos se dê também em razão do estilo de vida, fatores dietéticos ou por diferenças no acesso ao diagnóstico da doença (DAMIÃO et al., 2015).

A hereditariedade também apresenta importância. Se um parente de primeiro grau tem a doença, o risco é, no mínimo, duas vezes maior do indivíduo ter CaP. Se dois ou mais indivíduos da mesma família são afetados, o risco aumenta em cinco a 11 vezes. Porém, a hereditariedade não parece ser fator prognóstico importante ou influenciar negativamente a mortalidade relacionada ao CaP (DAMIÃO et al., 2015).

\section{MÉTODOS DE PREVENÇÃO: ANÁLISE DO PSA}

O CaP é uma doença que gera grande preocupação aos homens. Sua etiologia não é definida, porém, sabe-se que é multifatorial e sua incidência aumenta com o envelhecimento. A descoberta precoce deste câncer é fundamental para um tratamento com sucesso ou mais brando à saúde dos indivíduos. Para ajudar no diagnóstico precoce utiliza-se a análise de um biomarcador chamado (PSA) (ASCARI et al., 2014).

O PSA é um biomarcador tumoral desde 1987, é uma glicoproteína secretada pela próstata normal e doente. Sua utilização é de grande importância para a prevenção e o tratamento ao $\mathrm{CaP}$, porém é sensível a interferências em suas concentrações 
sanguíneas. Diversos estudos acharam interferências de situações de morbidade, situações diárias de stress, exercício físico e outros fatores que atuam sobre os valores séricos do PSA. Essas alterações nos exames clínicos podem levar a investigações mais invasivas, como a biópsia, aumentando a complexidade do atendimento e o risco a maiores complicações desnecessárias (MESQUISTA et al., 2018).

O toque retal, apesar de desconfortável e constrangedor, ainda constitui uma importante ferramenta no diagnóstico e estadiamento do CaP, já que cerca de $80 \%$ dos tumores encontram-se na zona periférica da glândula prostática. Em cerca de $18 \%$ dos pacientes, o CaP é detectado pelo toque retal, independentemente da concentração sérica de PSA (ASCARI et al., 2014).

O PSA ainda é o marcador mais empregado no rastreamento e no acompanhamento do CaP. Trata-se de uma proteína identificada no líquido seminal, produzido principalmente pelo tecido prostático, não sendo um marcador câncerespecífico. Um valor de PSA abaixo de 4,0 ng/mL é aceito como normal. Entretanto, outros fatores podem causar elevação de PSA - como prostatites, isquemias e infartos prostáticos, hiperplasia prostática benigna. O aumento do valor do PSA acima de 0,75 $\mathrm{ng} / \mathrm{mL}$ ao ano nos pacientes com PSA acima de $4,0 \mathrm{ng} / \mathrm{mL}$ parece estar associado a alto risco para CaP. Já nos pacientes com PSA inferior a 4,0 ng/mL, variações de $4 \mathrm{ng} / \mathrm{mL}$ podem ser consideradas significativas, principalmente nos mais jovens. $\mathrm{O}$ ajuste do valor do PSA de acordo com a idade tem como objetivo aumentar a sensibilidade da detecção de câncer nas camadas mais jovens da população e aumentar a especificidade nas mais idosas. Desta forma, optou-se por reduzir o ponto de corte de PSA para 2,5 ng/mL até a sexta década de vida (MESQUISTA et at., 2018).

Contudo, encontram-se diversas dificuldades para a sua prevenção, associadas a fatores como: (1) falta de informação da população; (2) crenças sobre o câncer e seu prognóstico; (3) preconceito contra o exame preventivo e (4) carência de rotinas nos serviços para a prevenção do CaP (MESQUISTA et at., 2018).

\section{RESULTADOS E DISCUSSÃO}


O CP é o tipo de neoplasia maligna mais prevalente entre os homens e figura como o sexto tipo mais comum no mundo. De acordo Mesquita et al. (2018), o número de casos novos de CP estimados no ano de 2008 para o Brasil foi de 49.530, o que corresponderia a um risco estimado de 52 casos novos para cada100 mil homens (NADER; ABDALLAH; SILVA, 2009).

No entanto, informações errôneas sobre o câncer de próstata faz com que muitos indivíduos não realizem o exame de rastreamento dificultando o diagnóstico precoce que em muitos casos possibilitaria a chance de cura além de permitir um tratamento menos agressivo e até mesmo mutilante (BACARIN; OLIVEIRA, 2018).

Estudos comprovam que o câncer de próstata se manifesta principalmente acima dos 50 anos de idade e, com o aumento da expectativa de vida, é considerado muito importante nos dados apresentados de mortalidade entre homens, representando um sério problema de saúde pública (BAROUKI, 2012).

A Lei 10.289, de 20 de setembro de 2001 institui o Programa Nacional de Controle do Câncer de Próstata, incluindo a campanha institucional nas redes de comunicação com intuito de prevenir o câncer de próstata através das orientações (BAROUKI, 2012).

Os fatores de risco para câncer de próstata são, na maioria, desconhecidos e inevitáveis. A grande maioria dos casos ocorre em homens com idade superior a 50 anos e naqueles com história de pai ou irmão com câncer de próstata. Sobre a história familiar como fator de riscos aumentam de 2,2 vezes quando um parente de $1^{\circ}$ grau (pai ou irmão) é acometido pelo problema, de 4,9 vezes quando dois parentes de $1^{\circ}$ grau são portadores do tumor e de 10,9 vezes quando três parentes de $1^{\circ}$ grau têm a doença (GOMES et al., 2008).

O PSA começou a ser utilizado para a detecção do CAP na década de 90. Esse método revolucionou o panorama da doença, gerando um aumento considerável no número de homens diagnosticados com CAP, por meio da indicação e realização de uma biópsia de próstata. Isso permitiu um diagnóstico precoce da doença e, teoricamente, aumentou as possibilidades de cura (LUDWIG et al., 2016). 
Porém a combinação entre PSA e toque retal, segundo Paiva; Motta; Griep (2011) e Gomes et al., (2008) é mais indicada visto que o primeiro exclusivamente falho de 30 a $40 \%$ dos diagnósticos, o segundo, exclusivamente, falha em $20 \%$ e a associação perde apenas $5 \%$ dos casos. Como consta no trabalho de Gomes et al. (2008) o toque retal sem a combinação do PSA, quando detectado já se encontram em estado avançado da doença.

A desintegração desses dois métodos de diagnóstico de acordo Santos (2012) os valores do PSA frequentemente resultam em falsos positivos $(80 \%$ dos resultados positivos são falsos-positivos). Estes resultados falsos resultam, por sua vez, em dano psicológico para o homem, incluindo a preocupação permanente sobre o carcinoma prostático. Homens que têm falsos-positivos são submetidos a mais exames complementares do que os que não obtêm esses resultados. Um terço dos homens biopsiados experimentam sensação dolorosa, febre, hemorragia, infecção e dificuldade urinária.

Em um estudo de caso relatado por Alves e Assis (2007) contou que, o exame de PSA do paciente encontrava-se na faixa de normalidade e teoricamente estaria descartada a presença de neoplasia, mesmo assim o paciente foi submetido a exame de toque retal, realização de exame de ultrassom transretal da próstata com biópsia. $\mathrm{O}$ achado anatomopatológico da peça cirúrgica confirmou a biópsia, com exérese total do tumor. Estima-se que $25 \%$ de pacientes com PSA normal, são portadores de câncer de próstata.

Semelhantemente, Bertoldo e Pletsch, (2007) calcula que 23\% dos homens com câncer de próstata apresentam valores de PSA total dentro da faixa de normalidade (igual ou menor do que $4 \mathrm{ng} / \mathrm{ml}$ ), no entanto mais da metade dos portadores de PSA acima de 4 não tem câncer. Por isso o toque retal é imprescindível, permitindo detectar tumores em pessoas com PSA normal.

De acordo Amorim et al. (2011) o antígeno prostático que é produzido pelas células epiteliais da próstata e não especificamente pela célula cancerosa, pode estar alterado em outras patologias e resultar na realização de biópsias desnecessárias. 
Mediante diagnóstico mais preciso, a Sociedade Americana de Cancerologia, preconiza para a detecção precoce do câncer em indivíduos sem sintomas o toque retal e o PSA séricos anuais a partir de 50 anos de idade. Através do toque retal e do PSA diminuiu a incidência de doença tardia com influência nas taxas de mortalidade 7,8 , na medida em que o câncer de próstata pode ser curável, desde que diagnosticado precocemente (MIRANDA et al., 2004).

Esta detecção e tratamento precoce prevenirem a progressão do câncer e o aparecimento de metástase, também é provável que sejam detectados tumores que teriam um crescimento muito lento e que não causariam problemas à saúde do homem (GOMES et al., 2008).

Assim sendo, a atenção farmacêutica dentro de uma unidade de oncologia abrange várias atividades além da administrativa como, por exemplo, a clínica onde o farmacêutico juntamente com outros profissionais analisa as prescrições avaliando o tratamento a ser realizado, dando ao paciente uma melhor qualidade de vida. Dentro da equipe multidisciplinar oncológica, realiza-se o acompanhamento e intervenções farmacêuticas favorecendo o uso racional de medicamentos e diminuição dos erros de medicações. Esses erros de medicação também podem ser diminuídos com criação de manuais de normas e procedimentos farmacêuticos (ALMEIDA et al., 2015).

Pode-se considerar também que este profissional tem conhecimento dos protocolos e as terapias de suporte utilizadas, pois é incumbido de analisar as bibliografias repassando as informações de fontes seguras à equipe multidisciplinar sobre a farmacocinética, farmacodinâmica, doses usuais, dose máxima, estabilidades medicamentosas e interações físicas e químicas para melhorar a qualidade das prescrições, além de estar sempre atualizado sobre as novas terapias lançadas nos mercado decorrentes do avanço da tecnologia (ALMEIDA et al., 2015).

Em estudo realizado por Reis et al., (2013) analisou as intervenções dos farmacêuticos clínicos em um hospital de ensino terciário do Brasil e constatou que é imprescindível a presença do farmacêutico no corpo clínico. Foi confirmado estatisticamente pelo estudo que 14,6\% das prescrições analisadas teve algum problema 
relacionado a medicamento (PRM) e que em sete das dez intervenções feitas nas prescrições que apresentaram algum problema foram benéficas.

A participação do farmacêutico no tratamento do $\mathrm{CaP}$ em uma unidade oncológica é imprescindível, pois além de dar informações sobre o medicamento este profissional tem o conhecimento necessário para contribuir na diminuição de erros de medicação e efeitos adversos contribuindo na escolha da melhor opção de tratamento a ser utilizado maximizando os feitos terapêuticos, diminuindo os colaterais e melhorando a qualidade de vida do paciente (REIS et al., 2013).

\section{CONSIDERAÇÕES FINAIS}

O envelhecimento da população mostra que a saúde precisa ser aprimorada, principalmente a saúde do homem. A falta de informação, medo e o preconceito aparecem como os principais fatores de impedimento ao exame do toque retal.

A educação em saúde é um fator externo importante para estimular a realização dos exames preventivos de câncer de próstata. A realização desses exames avalia a função prostática em homens, especialmente a partir de 40 anos, quando as lesões começam a ser mais frequentes. É importante ressaltar que assim como em diversos tipos de câncer, o diagnóstico precoce de alterações pode ser decisivo para a cura.

Com essa revisão pode-se analisar que o exame PSA é importantíssimo, porém não tanto quando associado ao toque retal e o exame de ultrassom transretal da próstata com biópsia, visto que o PSA é altamente sensível, porém não é câncer-específico e a maioria dos homens com PSA elevado não tem CAP.

\section{REFERÊNCIAS BIBLIOGRÁFICAS}

ALMEIDA, R. G. L. et al. O Manejo da Êmese em uma Unidade Oncológica: a Necessidade da Intervenção Farmacêutica em Tempo Real. Revista Brasileira de Cancerologia, v. 61, n. 2, p.115-121, 2015. 
ALVES, L.S.; ASSIS, B.P. Câncer de próstata com PSA normal. Revista Medica de Minas Gerais, v. 1,n. 3, p. 169-171, 2007.

AMORIM, V.M.S.L. et al. Fatores associados à realização dos exames de rastreamento para o câncer de próstata: um estudo de base populacional. Cad. Saúde Pública, Rio de Janeiro, v.27, n.2, p.347-356, fev. 2011.

ASCARI, R. A. et al. Prevalência de exames diagnósticos de câncer de próstata em comunidade rural. Cogitare Enfermagem, v. 19, n. 1, p. 89 - 93, jan/mar, 2014.

BACARIN, V. P.; OLIVEIRA, R. A. Mitos e medos no exame preventivo do câncer de próstata. Revista Olhar Científico, v. 04, n.1, p. 640, jan. /jul. 2018.

BAROUKI, M.P. Rastreamento do câncer de próstata em homens acima de 50 anos Através do exame diagnóstico de PSA. Revista Eletrônica Gestão \& Saúde. V.3, No. $2,2012$.

BERTOLDO, D.; PLETSCH, M.U. Estudo de prevalência de níveis de PSA encontrados em um município do interior do estado do Rio Grande do Sul. Revista Contexto e Saúde, Ijuí, v.7, n.13, p.33-40, jul. / dez. 2007.

BRASIL. INCA (Instituto Nacional do Câncer). Ministério da Saúde. Informativo Detecção Precoce. Boletim ano 5, n. 2 maios/agostos 2014. Disponível em: http://www1.inca.gov.br/inca/Arquivos/Informativo_D eteccao_Precoce_2_agosto_2014. Acesso em: 22 de abril de 2019.

DAMIÃO, R. Câncer de próstata. Revista HUPE, v. 14, n. 1, p.80-86, agosto, 2015.

FERNANDES, M. V. et al. Perfil epidemiológico do homem com câncer de próstata atendido em um hospital universitário. Cogitare Enfermagem, v. 19, n. 2, p. 333-340, abr/jun., 2014.

GOMES, R. et al. A prevenção do câncer de próstata: uma revisão da literatura. Ciência \& Saúde Coletiva, Rio de Janeiro v13,n.1, p.235-246, 2008.

GUEDES, G. A.; JÚNIOR, A. M. C. As Vantagens da Utilização do Exame Antígeno Prostático Específico (PSA) no Diagnóstico do Câncer de Próstata. Revista de Divulgação Científica Sena Aires, v. 1, p. 89 - 96, jan-jun, 2014.

LUDWIG, G.D. et al. Modelo preditivo integrado para a presença de câncer de próstata utilizando dados clínicos, laboratoriais e ultrassonográficos. Revista do Colégio

Brasileiro de Cirurgiões, Rio de Janeiro, v.43, n.6, nov./dez. 2016. 
MELO, W. A.; TEIXEIRA, M.; CARVALHO, M. B. Fatores demográficos associados à realização do antígeno. revista eletrônica gestão \& saúde. Revista Eletrônica Gestão \& Saúde, edição especial, p. 2078- 2086, março, 2013.

MESQUITA, J. V. M. et al. O autoconhecimento dos militares do sexo masculino sobre o câncer de próstata no município de Floriano-PI. Revista da FAESF, vol. 2, n. 1, p. 1 - 4, Jan-Mar. 2018.

MIRANDA, P. S. C. et al. Práticas de diagnóstico precoce de câncer de próstata entre professores da faculdade de medicina - UFMG. Rev Assoc Med Bras, v, 50, n. 3, p. 272- 275, 2004.

NADER MARTA,G.;HANNA ABDALLAH,S.; SILVA,J.L.F. PSA, toque retal e câncer de próstata. Rev.Fac.Ciênc.Méd. Sorocaba, v. 11, n. 2, p. 40 - 41, 2009.

PAIVA, E.P.; MOTTA, M.C.S.; GRIEP, R.H. Barreiras em relação aos exames de rastreamento do câncer de próstata. Rev. Latino-Am. Enfermagem, v.19, n. 1, jan./fev. 2011.

REIS, W. C. T. et al. Análise das intervenções de farmacêuticos clínicos em um hospital de ensino terciário do Brasil. Einstein, Curitiba, v. 11, n. 2, p.190-196, 2013.

RIBEIRO, L. S. et al. Conhecimento de homens acerca da prevenção do câncer de próstata. Revista Ciência Saúde, Nova Esperança, v. 13, n. 2, p.4 - 10, dez., 2015.

SANTOS, J.A. Hiperplasia prostática benigna e PSA: o efeito dominó. Rev bras med fam comunidade. Florianópolis, v. 7, n. 25, p. 259-64, Out./Dez., 2012 